\title{
Word recognition with forced serial processing: Effects of segment size and temporal order variation*
}

\author{
JEFFREY R. TRAVERS $\dagger$ \\ Swarthmore College, Swarthmore, Pennsylvania 19081
}

\begin{abstract}
Serial processing was forced by displaying words one letter or letter cluster at a time. Letters or clusters appeared in adjacent spatial positions and in rapid sequence, followed immediately by a mask. Under these conditions, there was a sharp increase in the percentage of words correctly identified as the size of the letter clusters presented in series increased. In a control condition without masking, designed to permit parallel processing across clusters, words were identified near-perfectly, regardless of the size of the clusters displayed. Words displayed one letter at a time without masking were identified fairly well even when letters were presented in random order. The results are interpreted as evidence that skilled readers tend to process letters within words in parallel.
\end{abstract}

Does the skilled reader process the letters within a word one at a time, in series, or does he process letter clusters or whole words in parallel? This deceptively simple question has proved difficult to answer. Introspection suggests that words, at least short words, are apprehended as wholes-but introspections about rapid, semiconscious processes can be unreliable. For example, the spoken word is an event which takes place over a period of hundreds of milliseconds, yet the time dimension is commonly lost in speech perception. Objective, functional evidence is required in order to determine whether the reader's subjective experience is as misleading as that of the speaker/hearer. Although existing findings are generally assumed to support the parallel processing hypothesis, several writers (e.g., Gough, 1972; Travers, 1970) have argued that most of the evidence is equivocal. More generally, Townsend (1972) has demonstrated that parallel and serial models are extremely difficult to separate experimentally; given sufficient flexibility in assumptions, both classes of model can generate identical predictions about performance in a variety of tasks.

The results of at least one recent study (Travers, 1973) can be explained most naturally in terms of parallel processing, although they do not rule out serial models on strictly logical grounds. Using a computer-controlled oscilloscope, Travers displayed common English words one letter at a time. In the conditions of interest here, letters appeared in normal adjacent spatial positions and in temporal order corresponding to their left-right sequence within the

*The research reported in this paper was supported by Regional Grant OEG-3-72-0050 from the Office of Education, U.S. Department of Health, Education and Welfare. Thanks are due to Robert Shriver, who served as a research assistant for both studies, and to Professor Edward Smith, who commented on the manuscrip $t$.

†Requests for reprints should be addressed to the author, c/o Deparment of Psychology, Swarthmore College, Swarthmore, Pennsylvania 19081 . word. In some cases, letters were followed immediately by masks, intended to interfere with iconic memory and thereby to force serial processing of individual letters. In other cases, words were simply painted left to right, without masking. This condition was designed to allow iconic memory to preserve initial letters as later ones appeared, thus permitting the $S$ to chunk and encode simultaneously the entire sequence, or relatively large letter clusters within the sequence. (See the "Serial Display" portion of Fig. 1, below, for further clarification of the display conditions.) The mask was found to impair word recognition markedly at brief exposure durations. At $50 \mathrm{msec}$ per letter, masked words were identified $58.4 \%$ of the time, and unmasked words, $84.2 \%$. In the masked condition, identification accuracy rose sharply as the time available for processing individual letters increased, reaching $85.0 \%$ at $200 \mathrm{msec}$ per letter. In contrast, word identification in the unmasked conditions was almost unaffected by letter exposure durations. (It rose from $84.2 \%$ to $87.0 \%$ as exposures increased from 50 to $200 \mathrm{msec})^{\mathbf{1}}$ These results were interpreted as showing (1) that serial display with masking does induce serial processing, as intended; (2) that accurate serial processing requires $150-200 \mathrm{msec}$ or more per letter, even when letters appear in common words; (3) most important, that serial processing is an inefficient and unnatural strategy for the skilled reader, who can recognize words at rapid exposure durations if allowed to make use of several letters simultaneously.

A second experiment was run (also reported in Travers, 1973) to rule out a possible alternative explanation and to amplify the above findings. The visual conditions of the first study were replicated, but random letter strings, rather than English words, were used as stimuli. The presence or absence of the mask was found to have no effect on recognition accuracy with such stimuli. Accuracy depended sharply upon letter exposure duration in both the masked and unmasked 

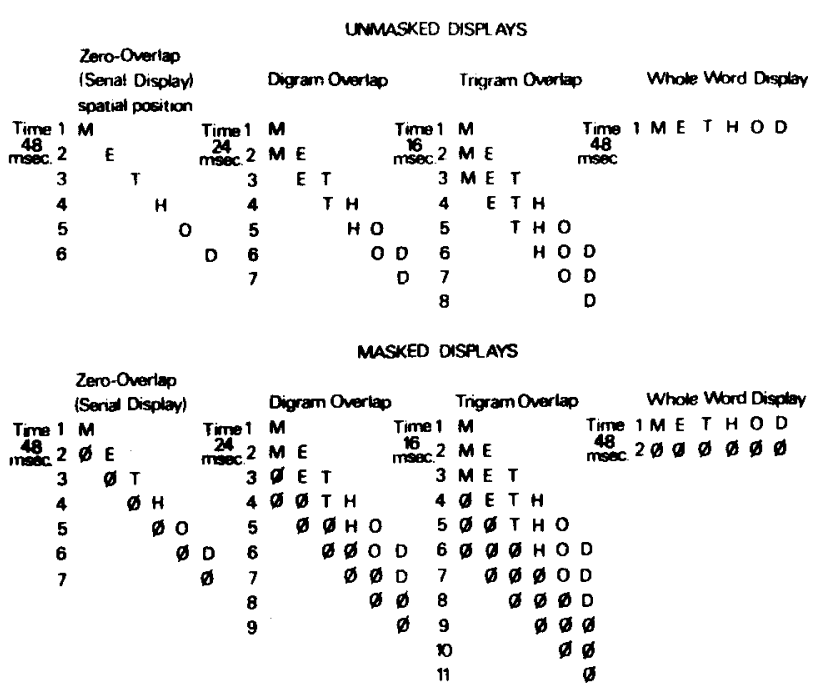

Fig. 1. Display conditions for Experiment I, temporal overlap study.

cases, and accuracy levels were nearly identical across masking conditions at each exposure duration. This experiment showed that (1) the effects of the mask in the previous experiment were indeed due to interference with the simultaneous encoding of letters and not merely to impairment in processing of individual letters, and (2) the advantage of parallel processing depends on Ss' knowledge about some property or properties of words (statistical redundancy, orthographic or phonological regularity, etc.) and is not due to general features of the visual system. Similar conclusions, based on a related experimental technique, have been reached by Manelis and Atkinson (in press). Manelis and Atkinson displayed two-syllable words as a sequence of syllables, with each syllable followed by a mask. There was a $50 \%$ overlap in time between the on-period for the first and second syllables. The investigators found that such quasi-serial displays made words harder to recognize than simultaneous presentation of both syllables. The effect was found not to apply to strings of unrelated characters-digits in their study.

The present paper reports two experiments designed to confirm and extend the methodological assumptions and substantive conclusions of the work described above.

\section{EXPERIMENT I TEMPORAL OVERLAP VARIATION}

In Experiment I, letters within words were shown with varying degrees of temporal overlap. That is, some words were shown one letter at a time, some two letters at a time, some three letters at a time, and some as wholes. For half of the words, the displayed segments were followed immediately by a mask; for the other half, no mask was used (see Fig. 1). According to the methodological assumptions underlying the earlier experiments, an increase in the number of letters present on the screen entails increased opportunity for parallel processing in the masked condition. In contrast, unmasked serial display is assumed to permit a substantial degree of parallel processing regardless of the number of letters displayed at any one time. If it is further assumed that skilled readers prefer to encode words in parallel, the following predictions can be made: (1) Increasing the degree of temporal overlap among letters should improve recognition accuracy in the masked condition; (2) the increase in temporal overlap should have little or no effect in the unmasked condition; and (3) for serial displays, the unmasked condition should produce better recognition than the masked condition, as in Travers (1973). For whole-word displays, the gap in accuracy between the masked and unmasked conditions should be smaller than in the serial case, since masked whole-word displays permit parallel processing scross the entire word. The gap should be of intermediate size for two- and three-letter clusters.

\section{Method}

Apparatus. The experiment was performed at the computer-based laboratory of the Harvard psychology department. Words were displayed on the cathode ray tube (CRT) of a Digital Equipment Corporation Type 340 precision display oscilloscope, controlled by a DEC PDP-4 computer. The CRT was equipped with P24 phosphors, which fade to $1 / 10$ of peak intensity in 1.5 microsec. The time required for a letter to reach peak intensity was about $3 \mathrm{msec}$, including both the time needed by the computer to process a display instruction and the time for the phosphors to respond. ${ }^{2}$ Thus, the apparatus provided fairly close control over stimulus duration; the total start-up and fade time was relatively small when compared to stimulus du rations $(48 \mathrm{msec})$.

Display programming was facilitated by use of Lexigraph, a language devised for experiments of this type by Daniel Forsyth. Lexigraph uses uppercase block characters formed by a pattern of closely spaced dots. At the letter sizes and exposure durations used in the present experiment, the dots were barely perceptible; characters essentially appeared to be formed by unbroken lines. Characters measured approximately $5 / 32 \times 7 / 32$ in. The visual angle subtended by characters varied, since Ss were allowed to adjust their viewing distance for comfort. Typical viewing distances were about 18-24 in. Characters appeared in luminescent green against a dark gray background. The mask, a zero crossed with a diagonal $(\phi)$, was roughly similar in size and brightness to letters.

Word Lists. Stimulus lists for all experiments were drawn from the Kucera and Francis (1967) count of one million words of printed English. All words used were common, with frequencies falling between 25 and 450 in the million-word sample. Word lists used in different experimental conditions had highly similar frequency distributions, with means not more than a few percentage points apart. Frequency distributions were also controlled across word lengths.

Experimental Conditions and Design. The following temporal overlap conditions were employed (see Fig. 1 for further clarification): (1) Zero overlap, or serial presentation: Words were printed across the CRT face one letter at a time, with letters in normal relative positions and in temporal order corresponding to normal left-right spatial sequence. (2) Bigram overlap: Words were printed from left to right, as in the first condition, but as a series of bigrams rather than single letters. The time span for each letter overlapped $50 \%$ with the preceding letter and $50 \%$ with the following letter. (3) Trigram overlap: 
Words were printed from left to right as a series of trigrams. Each letter overlapped $67 \%$ in time with the immediately preceding and following letters, and $33 \%$ in time with the letters removed by one additional letter position to the left and right. (4) Simultaneous, or whole-word, display: Words were shown with all letters present on the screen at once, in normal adjacent spatial positions.

The four types of display thus represent increasing degrees of temporal overlap among letters within words. Within each overlap condition, half of the words had a "zero" mask following each letter or letter cluster, and half had no mask.

The display time for each letter was kept constant at $48 \mathrm{msec}$. Therefore, increasing temporal overlap entailed a decrease in total processing time for the word. For example, a five-letter word displayed one letter at a time consumed $240 \mathrm{msec}$. A five-letter word displayed with bigram overlap required $144 \mathrm{msec}$, with trigram overlap $102 \mathrm{~m} \mathrm{sec}$, and with simultaneous display of all letters 48 msec. If ' $\mathrm{Ss}^{\prime}$ word-recognition performance improved with increasing overlap in the masked conditions, this fact would represent rather strong evidence for the utility of parallel processing (although, as noted earlier, it might still be possible to construct serial models to handle the data).

Each S identified a total of 200 words, 25 in each of the eight experimental conditions (four degrees of overlap, each with and without masking). Each block of 25 words included five words of each length from four to eight letters. Words of different lengths were presented in random order within conditions. Conditions were presented as blocks, and the order of blocks was randomized across Ss.

Procedure. Instructions to Ss were displayed on the CRT face. A research assistant was present at the beginning of the experiment to answer questions, but then left Ss alone. Ss were allowed to proceed at their own pace. They were asked to identify words as wholes if possible, but to record any letters they saw in cases where they were unable to identify the word. Identifications were recorded in writing by the Ss. (The recording pad was illuminated by a dim light. Otherwise, the experimental room was dark, and the face of the CRT was shielded from the light.)

Ss controlled the onset of stimuli by a button connected to the computer. After the $S$ pressed the button, a pair of colons (: :) appeared for $500 \mathrm{msec}$, bracketing the space in which the word was to appear. The colons served three purposes: (1) signaling the onset of the stimulus, (2) showing the $S$ where to focus his eyes, and (3) giving the $S$ some idea of how long the stimulus word would be. Before the block of trials for each experimental condition, Ss were given 10 practice trials to familiarize them with the forthcoming displays.

Subjects. Ss were 10 paid volunteers from Harvard and Radcliffe colleges. None reported reading disabilities or uncorrected vision defects.

\section{Results}

The results of the temporal overlap experiment are presented graphically in Fig. 2 . The same data, with an additional breakdown by word length, are given in Table 1.

When the mask was present, controlling the span of letters which could be processed at any one time, the outcome was a monotonic increase in accuracy of word recognition as that span increased. Only $33.5 \%$ of words presented one letter at a time were identified. In contrast, $84.4 \%$ of words displayed as wholes were identified. [It should be noted that this level of accuracy for masked whole-word displays at $48 \mathrm{msec}$ is considerably higher than levels which would be expected

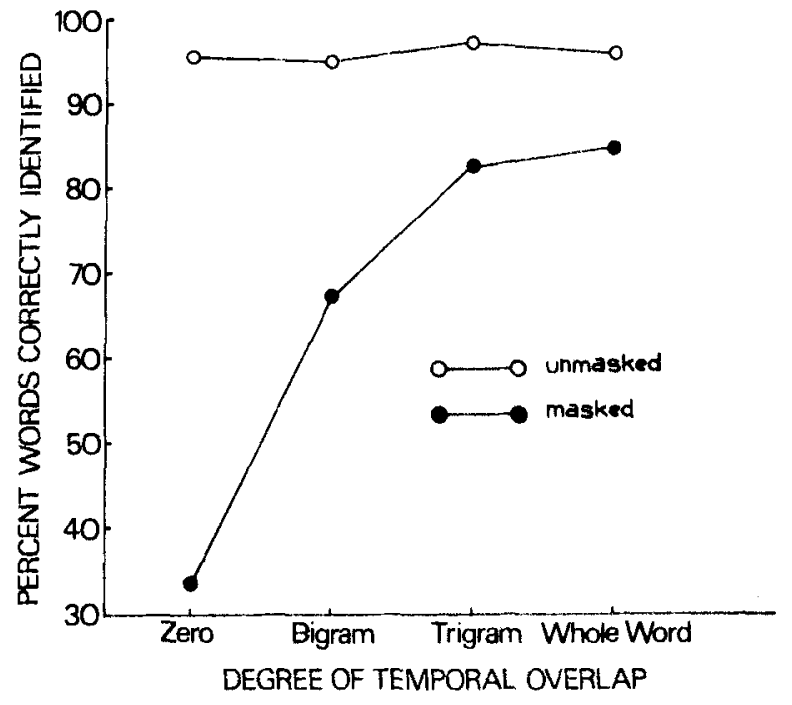

Fig. 2. Results of temporal over lap study: percentage of words correctly identified as a function of masking condition and degree of overlap.

on the basis of earlier reports, e.g., by Gilbert (1959) and by Scharf, Zamansky, and Brightbill (1966). The difference may be due to differences in the display apparatus and mask.] Bigram and trigram overlap produced intermediate results- $-67.2 \%$ and $82.4 \%$ correct identifications, respectively.

The data from the unmasked conditions show near-perfect performance regardless of the degree of overlap; identification levels varied from $94.8 \%$ to $96.8 \%$ across overlap conditions. Particularly striking is the fact that words displayed as wholes were recognized no better than words displayed one letter at a time.

A four-way analysis of variance was performed, using the number of words identified as the dependent variable. Masking condition, degree of overlap, and word length were treated as fixed independent variables; Ss were treated as a random independent variable. ${ }^{3}$ The main effects for masking and overlap were highly significant $(p<.001$ in both cases), as was that for length $(p<.02)$. The Masking by Overlap interaction was the only significant two-way interaction $(p<.001)$. The masking and overlap main effects accounted, respectively, for $28 \%$ and $14 \%$ of the total sum of squares, and their interaction for $12 \%$. As in earlier work, the length effect, though significant, accounted for only $1 \%$ of the total sum of squares and showed no consistent trend. Therefore, length effects will not be discussed further here (see Travers, 1973).

Following the significant $F$ test, individual differences among degrees of overlap within the masked condition were tested by the Newman-Keuls procedure (Winer, 1971, pp. 191-195). The serial display, or single-letter, condition proved to be significantly different from the other three $(p<.01$ in all cases). The differences between the bigram condition and the trigram and 
Table 1

Experiment I: Temporal Overlap Study. Number and Percent of Words Correctly Identified as a Function of Word Length, Degree of Overlap, and Masking Condition

\begin{tabular}{|c|c|c|c|c|c|c|c|c|c|c|}
\hline \multirow{3}{*}{$\begin{array}{c}\begin{array}{c}\text { Word } \\
\text { Length } \\
\text { (Letters) }\end{array} \\
\text { Unmasked }\end{array}$} & \multicolumn{8}{|c|}{ Degree of Overlap } & & \\
\hline & \multicolumn{2}{|c|}{$\begin{array}{c}\text { Serial } \\
\text { Display } \\
\text { No. Percent } \\
\end{array}$} & \multicolumn{2}{|c|}{ 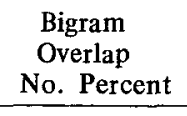 } & \multicolumn{2}{|c|}{$\begin{array}{l}\text { Trigram } \\
\text { Overlap } \\
\text { No. Percent } \\
\end{array}$} & \multicolumn{2}{|c|}{$\begin{array}{c}\text { Simultaneous } \\
\text { Display } \\
\text { No. Percent }\end{array}$} & \multicolumn{2}{|c|}{ Total } \\
\hline & & & & & & & & & & \\
\hline 4 & 48 & 96 & 50 & 100 & 48 & 96 & 48 & 96 & 194 & 97.0 \\
\hline 5 & 49 & 98 & 48 & 96 & 48 & 96 & 49 & 98 & 194 & 97.0 \\
\hline 6 & 49 & 98 & 50 & 100 & 47 & 94 & 50 & 100 & 196 & 98.0 \\
\hline 7 & 48 & 96 & 47 & 94 & 49 & 98 & 49 & 98 & 193 & 96.5 \\
\hline 8 & 44 & 88 & 42 & 84 & 50 & 100 & 46 & 92 & 182 & 91.0 \\
\hline Total & 238 & 95.2 & 237 & 94.8 & 242 & 96.8 & 242 & 96.8 & 959 & 95.9 \\
\hline \multicolumn{11}{|l|}{ Masked } \\
\hline 4 & 20 & 40 & 30 & 60 & 40 & 80 & 43 & 86 & 133 & 66.5 \\
\hline 5 & 18 & 36 & 32 & 64 & 42 & 84 & 44 & 88 & 136 & 68.0 \\
\hline 6 & 14 & $23^{*}$ & 40 & 80 & 49 & 98 & 43 & 86 & 146 & 73.0 \\
\hline 7 & 22 & 44 & 32 & 64 & 41 & 82 & 41 & 82 & 136 & 68.0 \\
\hline 8 & 13 & 26 & 34 & 68 & 34 & 68 & 40 & 80 & 121 & 60.5 \\
\hline Total & 87 & $33.5^{*}$ & 168 & 67.2 & 206 & 82.4 & 211 & 84.4 & 672 & 66.5 \\
\hline
\end{tabular}

*Due to an error in experimental procedure, $S$ s saw six items in the masked, serial six-letter condition.

whole-word conditions barely missed significance at the .05 level. The trigram/whole-word comparison was clearly nonsignificant.

Thus, when a mask is present, words are identified more accurately as the number of letters available for processing at any one time grows, even when increases in the letter span are associated with a marked decrease in processing time for the word as a whole. This finding is consistent with the hypothesis that parallel processing is the skilled reader's preferred strategy for dealing with words. However, the fact that the improvement in performance associated with increasing letter span diminishes after two letters and is negligible above spans of three letters suggests that Ss may not need to process whole words in parallel. The recognition system appears to hit peak efficiency when only three letters are available for simultaneous analysis, at least under present experimental conditions.

The high level of performance in the unmasked conditions and the weak association between identification accuracy and degree of overlap are consistent with the hypothesis that unmasked serial displays permit parallel processing regardless of the size of the chunks displayed. (However, for reasons stated in Note 1 , it is likely that increasing temporal overlap does permit some increase in parallel processing even when no mask is present. Apparently, this increase is either offset by the decrease in total processing time or obscured by ceiling effects.)

Comparison within the unmasked condition yields additional information on a point raised in response to the work of Travers (1973). Phillip Liss (personal communication) pointed out the fact that unmasked serial displays may not permit full parallel processing of longer words at most of the exposure durations employed (Note 1). Liss argued that incomplete parallel processing might account for the fact that average performance in the unmasked condition was only about $86 \%$ in that experiment. On the other hand, this less-than-perfect accuracy at rather slow display rates might be due to any of several other factors, including: (a) $\mathrm{S}$ fatigue (Ss identified 550 words in one session); (b) lack of skill or motivation on the part of the S group as a whole; (c) confusability of certain letters in the computer character set; (d) blinking, inefficient eye movements and apparent motion produced by the unusual visual displays.

Several observations from the present study cast doubt on Liss's conjecture that incomplete parallel processing due to loss of letters from iconic memory accounts for the low identification levels observed for unmasked serial displays in the earlier work. First, performance in the unmasked serial condition of the present study was substantially closer to perfect than had been the case earlier (about $96 \%$ as opposed to $86 \%$ ). This fact suggests that $\mathrm{S}$ fatigue, skill, or motivation, rather than incomplete parallel processing or any of the other explanations offered above, may have made the difference. (The present experiment was less than half as long as the previous one.) Second, performance in the unmasked condition was almost as good for serial as for simultaneous presentation (a test Liss had suggested). Finally, the fact that Ss in the masked condition did almost as well given three letters as they did given whole words suggests that partial parallel processing may be sufficient for the experimental task, and partial parallel processing was feasible with the serial displays of the previous study.

Performance in the masked serial condition of the present study was substantially worse than in the same condition of the earlier study ( $33 \%$ vs $58 \%$ correct). It is likely that this difference is due to the greater 
effectiveness of the "zero" mask than of the crosshatched number symbol (\#) used previously. (The differential effectivenss of the masks was confirmed in an auxiliary investigation.) This factor may also account for the nonzero difference between the masked and unmasked conditions even with simultaneous displays. The results with random strings reported by Travers (1973) led to the conclusion that the crosshatch mask does not interfere with individual letter recognition at display rates of $50 \mathrm{msec}$. If this conclusion held for the zero mask, it would be difficult to see why there should be any difference between masked and unmasked whole-word displays, since neither parallel processing nor individual item recognition should be impaired.

\section{EXPERIMENT II TEMPORAL ORDER VARIATION}

In Experiment II, Ss were shown words one letter at a time, with and without masks following each letter. Some words were shown with letters in normal positions and in temporal order corresponding to their left-right spatial sequence. Other words were shown with letters in normal position but in random temporal order. (See Fig. 3, below, for clarification of the display conditions.) The words used were short and the exposure durations brief, so that the entire display time fell within the span of iconic memory.

The unmasked condition of this experiment constitutes a stringent test of the hypothesis that iconic memory preserves temporally prior letters as later ones are being shown. If words can be read with a high degree of accuracy even when their separate letters are displayed in random order, this would be strong evidence that some mechanism must be operating to preserve those letters in spatial, rather than temporal, arrangement. In fact, if we take literally the hypotheses that (a) iconic memory preserves perfectly the information in unmasked, random-order displays, and (b) Ss invariably process unmasked serial displays by postponing encoding until the entire display is present in iconic memory, we are forced to predict that identification will be as accurate for random-order displays as for normal-order displays in the unmasked condition.

A different prediction seems appropriate for the masked condition. If $\mathrm{Ss}$ are forced by the mask to encode letters in the temporal order of the display, random-order displays will produce encoding which conflicts with the correct order of letters within the word. The $\mathrm{S}$ may be able to unscramble the order of letters after the fact. And he may be assisted in this anagram strategy by whatever information he is able to retain concerning the spatial position of the letters in the original display. (It is not clear to what degree, if at all, masking interferes with retention of position information.) However, it seems reasonable to predict that the conflict between temporal order and spatial position will lower identification accuracy relative to that in the masked, normal-order condition. The $\mathrm{S}$ is likely to be confused by the fact that several words can often be formed from the same set of letters (e.g., SPOT, STOP, POTS, POST, TOPS, OPTS). Also, when letters are in order, previously processed letters can help the $S$ identify the next one to come and to guess at missed letters. When the $S$ must distinguish temporal from spatial order during a rapid display, such facilitation is likely to be minimal. In light of these considerations, it seems apparent that the effects of random vs normal order should be greater in the masked than in the unmasked case, even if the prediction of zero difference in the unmasked case is not met.

\section{Method}

The general procedure and apparatus were the same as in Experiment I, except that the oscilloscope was a Fairchild Type 737 A large-screen indicator, slaved to the 340 scope used in the previous study. Ten Ss were run, again paid volunteers from Harvard and Radcliffe. One reported a minor vision defect, but his performance was similar to that of the rest of the $S$ group.

Words were displayed one letter at a time at exposure durations of $50 \mathrm{msec}$ per letter. All of the words used were short-three to five letters. This restriction was adopted in order to guarantee that information from all letters would be available in iconic memory at once. In addition, the restriction allowed control over the possible effects of eye movements; when displays sweep left to right in conventional temporal order, eye movements tend to follow in the correct direction. When displays hop randomly from letter position to letter position, however, the eye movements provoked by the first few letters are unlikely to guide the eyes to an optimal point of focus for later letters. If a $\mathbf{S}$ moved his eyes during a randomly ordered display, he might easily $m$ iss some of the later letters. There would be no way to guarantee that all letters registered in iconic memory or to equate the effects of eye movements between random and conventionally ordered displays. However, the average latency for an eye movement is just under $200 \mathrm{msec}$ (Woodwor th \& Schlosberg, 1954, p. 502). Therefore, by keeping the total display time for a word under $200 \mathrm{msec}$, it was possible to restrict eye movements during the display. At exposure durations of $50 \mathrm{msec}$, this required limiting the length of words to four letters or less. Of course, eye-movement latencies must be somewhat variable. More movements should occur with four-letter words than with three-letter words, and therefore we might expect somewhat worse performance with the longer words. (Five-letter words, which require $250 \mathrm{msec}$ to display, exceeding the average eye-movement latency, were included as a check on this general line of reasoning. If eye movements operate in the manner described, we might expect a relatively sharp drop in recognition accuracy as words increase in length from four to five letters in the unmasked condition. For this reason, five-letter words are excluded from most of the analyses described below.) It was not desirable to lower the 50 -msec letter exposure duration because Mayzner and his associates (e.g., Mayzner, Tresselt, \& Cohen, 1966) have shown that serial displays at faster rates produce a curious perceptual effect ("sequential blanking") in which some letters simply disappear subjectively.

Ss identified a total of 288 words, 72 with masks and 216 without. One-third of the words in each condition (24 and 72 words, respectively) were three letters long, one-third four letters long, and one-third five letters long. Words were displayed one letter at a time, with letters in proper spatial position (see Fig. 3). The temporal order in which letters were presented was 


\section{NORMAL ORDER}

UNMASKED

Spatial Position

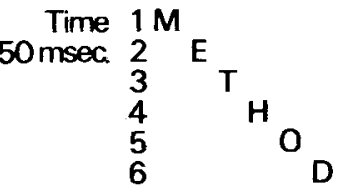

RANDOM ORDER

MASKED

Spatial Position

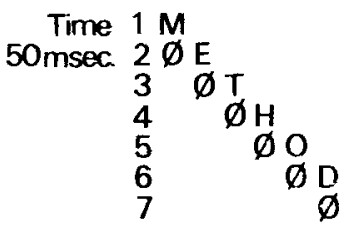

UNMASKED

Spatial Position

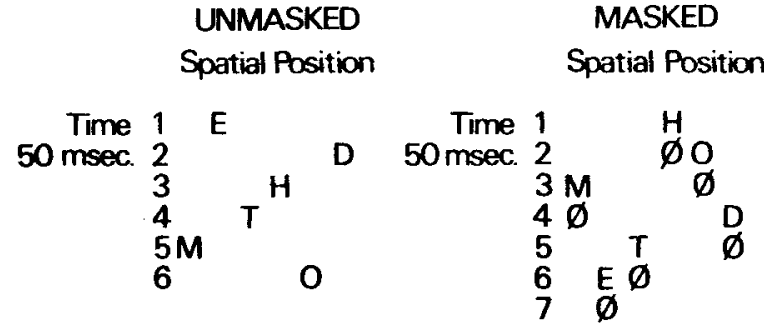

Fig. 3. Display conditions for Experiment II, temporal order variation study.

determined as follows: In the case of three- and four-letter words, all possible orders were used. Each of the six possible orderings of three-letter words appeared 4 times in the block of masked displays and 12 times in the unmasked displays. Each of the 24 possible orderings of four-letter words appeared once in the masked displays and 3 times in the unmasked. In the case of five-letter words, which have 120 possible orderings, random selection was used to determine the orderings in both the masked and unmasked conditions. The presentation order of the different temporal orderings and word lengths was random. Masked and unmasked words were presented as blocks; half of the Ss saw the masked block first, half the unmasked.

The procedures for selecting temporal orders of presentation guaranteed, at least in the case of three- and four-letter words, that a subset of words would be displayed in normal temporal order, i.e., an order corresponding to the left-right spatial arrangement of letters. Sixteen three-letter words ( 4 masked, 12 unm asked) and 4 four-letter words ( 3 unmasked, 1 masked) were shown in normal order. In addition, random selection dictated that 2 five-letter words, both unmasked, would also be shown in normal order. Thus, the experiment incorporated a limited internal comparison of the effects of random vs normal ordering, as well as providing data that could be compared with other studies in which strictly normal ordering was used.

\section{Results}

Table 2 gives the results of the temporal order variation study. The number and percentage of words correctly identified by $10 \mathrm{Ss}$ are shown as a function of masking condition, normal vs random temporal order, and word length. Several findings emerge from the data:

(1) In the absence of a mask, words were identified with a relatively high degree of accuracy (an average of $80.5 \%$ for three- and four-letter words), even when displayed with letters in random order. Ss were able to preserve and reorder letters, either through direct storage of position information or through unscrambling words after the display terminated. ${ }^{4}$

(2) When a mask was present, only $27.7 \%$ of randomly ordered three- and four-letter words were identified. It seems fair to assume that the S's ability to unscramble words is the same across masking conditions, given equal accuracy in identifying individual letters. Moreover, although Experiment I indicated that the mask might interfere somewhat with individual letter identification, the effect was small relative to the very large difference between the masked and unmasked random-order condition in Experiment II. Therefore, it is reasonable to conclude that the difference is due to the operation of iconic memory, or some other form of storage mechanism, rather than to a difference in the conscious process of anagram solution after the display. It should be mentioned, however, that Ss varied in reported strategies for dealing with displays of this type. While none reported an experience of visual simultaneity of letters, several reported "taking in" the entire display visually and identifying the words as wholes. Others reported an attempt to identify individual letters and unscramble their order.

(3) When unmasked words were presented in normal order, levels of identification were very high, comparable to the levels obtained for similar displays in Experiment I ( $94.7 \%$ for three- and four-letter words). The $14.2 \%$ difference between normal and random unmasked displays was significant $(t=7.42 ; \mathrm{p}<.001)$. The joint hypothesis that iconic memory preserves letters perfectly, regardless of input order, and that Ss invariably wait to encode entire strings from iconic memory, was disconfirmed. Clearly, such a hypothesis may fail because of errors in either or both of its two assumptions, and in this case errors can be found in both. On the one hand, iconic memory is presumably not an all-or-none storage system but a decaying trace of some kind; thus, it is likely that some information is lost from iconic memory even within the brief display durations used in this study. On the other hand, as

Table 2

Experiment II: Temporal Order Variation Study. Number and Percentage of Words Correctly Identified as a Function of Length, Masking Condition, and Presentation Order of Letters

\begin{tabular}{llrrrr}
\hline & & Normal Order & \multicolumn{2}{r}{ Random Order } \\
Letters & & No. Percent & No. & Percent \\
\hline \multicolumn{5}{c}{ Masked } \\
3 & & 18 & 45.0 & 71 & 35.5 \\
4 & & 1 & 10.0 & 48 & 20.9 \\
& & 19 & 38.0 & 119 & 27.7 \\
5 & Subtotal & - & - & 20 & 8.3 \\
& Total & 19 & 38.0 & 139 & 20.7 \\
& & Unmasked & & \\
3 & & 113 & 94.2 & 486 & 81.0 \\
4 & & 29 & 96.7 & 553 & 80.1 \\
& & 142 & 94.7 & 1039 & 80.5 \\
5 & Subtotal & 20 & 100.0 & 477 & 68.1 \\
& Total & 162 & 95.3 & 1516 & 76.1 \\
\hline
\end{tabular}


indicated by introspective reports of Ss (Point 2, above) some Ss encode letters serially, despite the opportunity for parallel processing in the unmasked conditions.

(4) When masked words were presented in normal order, levels of identification were roughly similar to those obtained for masked serial displays in Experiment I (38.0\% vs $33.5 \%)$. The $10.3 \%$ difference in accuracy between the normal and random masked conditions missed significance $(t=1.63 ; 0.5<p<.1)$. The prediction that randomization of input order would be more damaging for masked than for unmasked displays was disconfirmed. The explanation for this failure is not immediately apparent; however, one possibility is the existence of a kind of floor effect in the masked case: With masking, Ss presumably process letters individually, whether presented in normal or random order, perhaps recognizing words as such only after the individual letters are spelled out. In the random-order case, this requires postperceptual rearrangement of letters. But this anagram strategy may be very effective with short words; there may be no way to force identification accuracy below 20\% without deliberately interfering with perceptual processing, e.g., by cutting premask exposure durations. The difference between the masked normal and random conditions might then show up more clearly with a reaction time measurement, which should be sensitive to the time consumed by the anagram stage of processing.

(5) When identification levels were broken down by word length, different patterns emerged in the masked and unmasked conditions. In the unmasked random condition, accuracy was the same for three- and four-letter words $(81.0 \%$ and $80.1 \%)$. Five-letter words were identified somewhat less well $(68.1 \%)$. The difference between the five-letter score and the pooled three- and four-letter scores was significant $(t=4.52$; $p<.001)$. This difference probably represents the effect of eye movements, as described earlier. In the masked random condition, three-letter words were identified markedly better than four-letter words, and four-letter words better than five-letter words $(37.1 \%, 20.4 \%$, and $8.3 \%$, respectively). This difference may be due to the fact that unscrambling short words is easier than unscrambling long ones. In the unmasked normal condition, length appeared to have almost no effect. Data for the masked normal condition were too meager to permit any conclusions.

In sum, the temporal order variation study confirmed the expectation that iconic memory could preserve input letters in spatial arrangement, despite random temporal order of input. However, the study did not support several more specific predictions derived from additional assumptions discussed in the introduction to this section of the report. Judgment must be reserved on the question of whether these failures are due to errors in the subsidiary assumptions, as suggested immediately above, or whether they point to more serious defects in the approach.

\section{DISCUSSION}

Specific conclusions relevant to the two studies have been stated above and need not be reiterated here. However, it is important to inquire whether we may draw any general lessons which set constraints upon possible models of word recognition. As noted in the introduction, Townsend (1972) has demonstrated mathematically that serial and parallel models can generally be made to "mimic" one another, i.e., that almost any given distribution of reaction times, accuracies, etc., can be "predicted" by some model in each class. (Of course, a specific serial or parallel model might be disconfirmed by some set of data; however, the general class of models could rarely be rejected or supported unambiguously.) As cases in point, consider the following examples:

(1) Gough (1972) has proposed a model of word recognition which incorporates rapid left-to-right serial processing of letters. His most direct evidence for serial processing concerns the effects of word length on word-naming latencies and latencies for word/nonword decisions. Since latencies in both cases increase with word length, Gough argues that serial processing of individual letters must be operating. However, it can be shown that a limited-capacity parallel-processing model can handle Gough's data.

(2) Travers (1973) interpreted his results on forced serial processing as evidence for a parallel model. Since word recognition was impaired by forced left-to-right serial processing, he concluded that parallel processing was the skilled reader's preferred strategy. However, Travers's displays did not merely force serial processing; they forced it in a given order and at a given fixed rate per letter. If readers in fact process in series, but in some order other than left to right, or if they prefer to allocate different amounts of time to different letter positions, Travers's serial displays would impair recognition for reasons other than the one he proposed. ${ }^{5}$

It is unlikely that any word-recognition experiment will yield data sufficiently precise to meet Townsend's rigorous criteria for separating parallel and serial model in general. Failing such a crucial test of the two classes of model, we can only test specific models and try to discover which class seems to fit the broader range of data and to use the more natural assumptions.

The present studies, in the opinion of the author, add credence to the parallel class of models. In Experiment I, it was shown that simultaneous presence of letters in iconic memory dramatically facilitated word recognition, even when simultaneous displays entailed equally dramatic reductions in the total time available for processing words. In Experiment II, it was shown that words could be read fairly well even though individual letters were displayed in random order. If Ss were processing letters serially, random-order displays should have impaired word recognition severely, as they did when visual conditions were such that serial 
processing was forced. It remains to be seen whether any serial model can give a plausible account of these results.

\section{REFERENCES}

Clark, H. H. The language-as-fixed-effect fallacy: A critique of language statistics in psychological research. Journal of Verbal Learning \& Verbal Behavior, 1973, 12, 335-359.

Gilbert, L. C. Influence of interfering stimuli on perception of meaningful material. California Journal of Educational Research, 1959, 10, 15-23.

Gough, P. B. One second of reading. In J. F. Kavanaugh and I. G. Mattingly (Eds.), Language by ear and by eye. Cambridge: M.I.T. Press, 1972 .

Haber, R. N., \& Standing, L. G. Direct measures of short term visual storage. Quarterly Journal of Experimental Psychology, $1969,21,43-54$

Kučera, H., \& Francis, W. N. Computational analysis of present-day American English. Providence, R.I: Brown University Press, 1967.

Manelis, L., \& Atkinson, R. C. Tachistoscopic recognition of syllabicated words. Quarterly Journal of Experimental Psychology, in press.

Mayzner, M. S., Tresselt, M. E., \& Cohen, A. Preliminary findings on some effects of very fast sequential input rates on perception. Psychonomic Science, 1966, 6, 513-514.

Scharf, B., Zamansky, H. S., \& Brightbill, R. F. Word recognition with masking. Perception \& Psychophysics, 1966, 1, 110-112.

Sperling, G. A model for visual memory tasks. Human Factors, $1963,5,19-31$.

Townsend, J. T. Some results concerning the identifiability of parallel and serial processes. British Journal of Mathematical \& Statistical Psychology, 1972, 25, 168-199.

Travers, J. R. Sequential and parallel processes in reading. Unpublished doctoral dissertation, Harvard University, 1970.

Travers, J. R. The effects of forced serial processing on identification of words and random letter strings. Cognitive Psychology, 1973, 5, 109-137.

Woodworth, R. S., \& Schlosberg, H. Experimental psychology. (Rev. ed.) New York: Holt, Rinehart \&z Winston, 1954.

Winer, B. J. Statistical principles in experimental design. (2nd ed.) New York: McGraw-Hill, 1971.

\section{NOTES}

1. In the original draft of Travers's (1973) paper, this result was interpreted as showing that the unmasked condition allowed full parallel processing across the entire word. However, Phillip
Liss (perso nal communication) pointed out that, if we accept the common estimate that iconic memory lasts about $250 \mathrm{msec}$ (e.g. Haber \& Standing, 1969), full parallel processing would not be possible at most exposure durations and word lengths employed in the experiment. What seems to occur is a trad eoff between total processing time and the opportunity for parallel processing: The longer the letter exposure duration, the more likely that the iconic trace of initial letters will have faded by the time later letters appear. On theother hand, the longer the exposure, the greater the total amount of processing time for the word. Presumably, both greater processing time and the opportunity for parallel processing facilitate word recognition. The two effects appear to have cancelled each other almost exactly. It is also possible, of course, that visual persistence is considerably longer than 250 msec for light-on-dark displays of the type used in Travers (1973) and in the present study. Sperling (1963), for example, has reported icon durations up to several seconds under these conditions.

2. The start-up time was incorrectly reported as $900 \mathrm{microsec}$ in Travers (1973). Thanks are due to Ronald Kaplan for correcting the misimpression given by Lexigraph's technical specifications.

3. In light of the arguments of Clark (1973), it must be pointed out that this analy sis treats words as a fixed effect. That is, the reported significance levels give us reason to believe that the results would replicate with new $S$ s randomly drawn from the same population, but no basis for concluding that they would hold with a new group of stimulus words.

4. A serious question may be raised as to whether these results are unique to light-on-dark displays, which produce far greater visual persistence than dark-on-light displays. While no direct information is available on this point, it can be mentioned that the results of Travers (1973) were first obtained in pilot studies using black-on-white displays presented via movies.

5. These two counterexplanations are offered only to illustrate the general point that single experiments are usually ambiguous with respect to the serial vs parallel processing issue. In fact, both explanations are probably wrong. As shown in Experiment II, serial processing in orders other than left-right tends to produce worse, not better, word recognition. Also, in pilot studies, it has been found that variations in the allocation of processing time across letter positions do not affect recognition accuracy. Neither set of data, of course, is conclusive.
(Received for publication January 25, 1974; revision received February $18,1974$. ) 